\title{
Steady State Response at the Interface of Elastic Half Space and Micropolar Liquid-saturated Porous Half Space
}

\author{
Praveen Ailawalia* \\ Department of Applied Sciences, Baddi University of Emerging Sciences and Technology, Makhnumajra, Baddi, District Solan, H.P., \\ 173205, India
}

Received: 24 May. 2013, Revised: 28 Sep. 2013, Accepted: 29 Sep. 2013

Published online: 1 May. 2014

\begin{abstract}
The steady state response of a micropolar liquid saturated porous solid with an overlying elastic half space to a moving point load along the interface has been investigated. The transformed components of displacement, microrotation, force stress (in solid and liquid parts) and couple stress are obtained by using Fourier transformation. These components are then inverted and the results are obtained in the physical domain by applying a numerical inversion technique. The numerical results are depicted graphically for a particular model. Some particular results are also deduced from the present investigation and these deduced results are compared with the already established results by previous researchers.
\end{abstract}

Keywords: Steady state, Micropoar, Liquid saturated porous, microrotation, fourier transformation

\section{Introduction}

The theory of micropolar continua was initiated by Eringen and Suhubi [5] and Suhubi and Eringen [6] as a special case of their work on micro-elastic solid, and was renamed couple stress theory. Later, Eringen [7] recapitulated and renamed it as micropolar theory. A similar theory appeared to be developed independently by Palmov [29] for the linear elastic solid. Physically speaking, the theory of micropolar elasticity is concerned with those materials whose constituents are dumbbell molecules. These elements are allowed to rotate independently without stretch. The basic difference between the theory of micropolar elasticity and that of classical elasticity is the introduction of an independent microrotation vector. In classical elasticity, all other quantities can be obtained from the knowledge of three components of the displacement vector. In micropolar elasticity, we must also have knowledge of the three components of microrotation vector. In micropolar elastic bodies, the force at a point of a surface element is completely characterized by a stress vector and a couple stress vector at that point, while in classical elastic theory, the effect of couple stress is neglected.
Physically, solids that are composed of dumbbell molecules may be adequately represented by the model of micropolar elasticity. Fibrous materials and some granular and porous bodies may also fall in the category of this theory (Eringen [7]). It is believed that "porous granular" material can be best approximated to soil (Deresiewicz [2]). Thus, a peculiar type of soil/rocks whose molecules are granular, e.g., polycrystalline material, aluminum-epoxy, concrete, may be examples of micropolar solids.

The dynamical response of solid material subjected to moving loads is of great interest to a number of engineering fields, such as civil engineering, ocean engineering, earthquake engineering and tribology. For example ground motion and stresses are induced in saturated soils by fast moving vehicular loads or surface blast waves due to explosives.

Various researchers investigated the dynamic response of half space subjected to a moving point load. Sneddon [33] was the first to discuss the two dimensional problem of a line load moving with constant sub-sonic speed over the surface of a homogenous elastic half space. Some of the similar problems of the sub-sonic, transonic and supersonic were discussed by other researchers (Cole

\footnotetext{
*Corresponding author e-mail: praveen_2117@ rediffmail.com
} 
and Huth [1]; Fung [11]; Fryba [10]. A homogenous three dimensional elastic half space subjected to forces moving with a constant speed was studied by Eason [4] using the double Fourier transformation method. Payton [30] considered the transient problem for a line load applied suddenly and then moving with a constant speed on the surface of an elastic half space. In micropolar theory of elasticity the steady state response to moving loads in a semi-infinite medium has been investigated by Sengupta and Ghosh [32]. Ghosh [13] discussed the steady state response to the applied load moving with constant speed for infinitely long time over the free surface of semi-space composed of a homogenous micropolar elastic solid layer on the top of a micropolar elastic solid medium of infinite extent. Kumar and his coworkers (Kumar and Gogna [16]; Kumar and Deswal [18,20]; Kumar and Ailawalia [21,22, 23,24,25] studied different types of moving load problems in the theory of micropolar elasticity.

It is believed that some soils whose molecules are granular, are very close to micropolar elastic porous medium. Hence, the present model is the motivation of the situation, when an elastic half space is resting on micropolar liquid-saturated porous foundation. Kumar and Miglani [17] studied the effect of pore alignment on surface wave propagation in a liquid-saturated porous layer lying on a liquid-saturated porous half-space with loosely bonded interface. Murad and Cushman [28] studied thermomechanical theories for swelling porous media with microstructure. Deswal et al. [3] discussed the effect of fluid viscosity on wave propagation in a cylindrical bore in micropolar elastic medium. Kumar and Deswal [19] studied wave propagation in micropolar liquid-saturated porous solid. Kumar and Barak [26] studied the reflection and transmission of plane waves at an interface between homogenous invisicid liquid half space and micropolar liquid saturated porous solid half space.

In the present investigation we have derived the expressions of displacement, microrotation, force stress and couple stress in micropolar liquid saturated porous medium with an overlying elastic half-space due to a moving point load along the interface by using Fourier transformation. Such types of moving load problems are quite important in many dynamical systems. Some of the results established by earlier researchers have also been deduced from the present investigation.

\section{Formulation and Solution of Problem}

We consider a normal point load along the interface of elastic half space (Medium II) /micropolar liquid saturated porous half space(Medium I). A rectangular coordinate system ' $x, y, z^{\prime}$ having origin on the surface $z=0$ and $z$-axis pointing vertically into the medium is considered as shown in figure 1 . We assume a pressure pulse $P(x+U t)$ which is moving with a constant velocity $U$ in the negative direction. Since the load has a

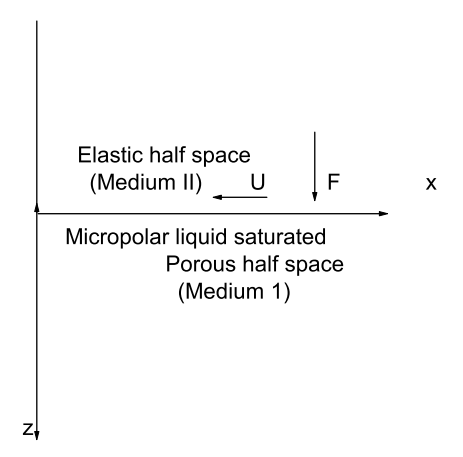

Fig. 1 Moving load along the interface.

constant magnitude and move with a constant speed, after a sufficiently long time the solid response may become stationary in the reference system that is fixed to the load. In this paper we study possible pattern of this stationary response.

\section{Basic Equations}

Following Eringen [7] and Konczak [14,15] the field equations and constitutive relations in micropolar liquid saturated porous solid(Medium I) in the presence of dissipation are given by,

$$
\begin{aligned}
(\lambda+2 \mu & +K) \nabla(\nabla \cdot \vec{u})-(\mu+K) \nabla \\
& \times(\nabla \times \vec{u})+K(\nabla \times \vec{\phi})+Q \nabla(\nabla \cdot \vec{w}) \\
= & \frac{\partial^{2}}{\partial t^{2}}\left(\rho_{11} \vec{u}+\rho_{12} \vec{w}\right) \\
& \quad+b \frac{\partial}{\partial t}(\vec{u}-\vec{w}) \\
\nabla(Q e+R \epsilon)= & \frac{\partial^{2}}{\partial t^{2}}\left(\rho_{12} \vec{u}+\rho_{22} \vec{w}\right) \\
\quad-b \frac{\partial}{\partial t}(\vec{u}-\vec{w}) & \\
(\alpha+\beta+\gamma) & \nabla(\nabla \cdot \vec{\phi})-\gamma \nabla \times(\nabla \times \vec{\phi}) \\
& +K(\nabla \times \vec{u})-2 K \vec{\phi}=\rho j \frac{\partial^{2} \vec{\phi}}{\partial t^{2}} \\
t_{k l} & =\left(\lambda u_{r, r}+Q w_{r, r}\right) \delta_{k l}+\mu\left(u_{k, l}+u_{l, k}\right) \\
& +K\left(u_{l, k}-\epsilon_{k l r} \phi_{r}\right) \\
m_{k l} & =\alpha \phi_{r, r} \delta_{k l}+\beta \phi_{k, l}+\gamma \phi_{l, k} \\
\sigma & =Q e+R \epsilon
\end{aligned}
$$

where $\lambda, \mu, K, \alpha, \beta, \gamma$ are material constants, $\rho$ is the density of micropolar elastic solid, $\jmath$ is microintertia, $\vec{u}$ and $\vec{w}$ are displacement vectors in solid and liquid parts respectively and $e=\operatorname{div} \vec{u}, \epsilon=\operatorname{div} \vec{w}$ are the 
corresponding dilatation; $\vec{\phi}$ is microrotation vector, $Q$ is a measure of coupling between the volume change of the solid and of the liquid, $R$ is a measure of the pressure that must be exerted on the fluid to force a given volume of it into the aggregate while total volume remains constant. $\rho_{11}, \rho_{12}, \rho_{22}$ are dynamical co-efficients and $b$ is a dissipation function, $\nabla$ is the gradient operator, $t_{k l}$ and $m_{k l}$ are respectively force stress tensor and couple stress tensor in medium I.

The equation of motion and stress- strain relation for an elastic medium are given by Ewing, Jardetzky and Press [8] as,

$$
\begin{aligned}
\left(\lambda^{e}+\mu^{e}\right) \nabla(\nabla \cdot \vec{v})+\mu^{e} \nabla^{2} \vec{v} & =\rho^{e} \frac{\partial^{2} \vec{v}}{\partial t^{2}}, \\
t_{i j}^{e} & =\lambda^{e} \theta \delta_{i j}+2 \mu^{e} e_{i j},
\end{aligned}
$$

where,

$$
\theta=v_{1,1}+v_{2,2}+v_{3,3}, \quad e_{i j}=\frac{\left(v_{i, j}+v_{j, i}\right)}{2},
$$

$\lambda^{e}, \mu^{e}$ are Lame's constant in elastic medium.

\section{Solution of Equations}

For two dimensional problem, all quantities depend only on space coordinates and time and we take the displacement vector and microrotation vector in medium I as ,

$$
\begin{aligned}
& \vec{u}=\left(u_{1}, 0, u_{3}\right), \\
& \vec{\phi}=\left(0, \phi_{2}, 0\right), \\
& \vec{w}=\left(w_{1}, 0, w_{3}\right) .
\end{aligned}
$$

The displacement components $u_{1}, u_{3}$ (solid part) and $w_{1}, w_{3}$ (liquid part) in medium I are related by potential functions $q, \psi$ and $H$ as,

$$
\begin{aligned}
u_{1} & =\frac{\partial q}{\partial x}+\frac{\partial H}{\partial z}, & u_{3} & =\frac{\partial q}{\partial z}-\frac{\partial H}{\partial x}, \\
w_{1} & =\frac{\partial \psi}{\partial x}, & w_{3} & =\frac{\partial \psi}{\partial z} .
\end{aligned}
$$

Using (10)-(11) in equations (1)-(3), we obtain,

$$
\begin{gathered}
{\left[\nabla^{2}-\frac{b}{(\lambda+2 \mu+K)} \frac{\partial}{\partial t}-\frac{\rho_{11}}{(\lambda+2 \mu+K)} \frac{\partial^{2}}{\partial t^{2}}\right] q} \\
+\left[\frac{Q}{(\lambda+2 \mu+K)} \nabla^{2}+\frac{b}{(\lambda+2 \mu+K)} \frac{\partial}{\partial t}\right. \\
\left.\frac{\rho_{12}}{(\lambda+2 \mu+K)} \frac{\partial^{2}}{\partial t^{2}}\right] \psi=0
\end{gathered}
$$

$$
\begin{aligned}
& {\left[\nabla^{2}+\frac{b}{Q} \frac{\partial}{\partial t}-\frac{\rho_{12}}{Q} \frac{\partial^{2}}{\partial t^{2}}\right] q} \\
& +\left[\frac{R}{Q} \nabla^{2}-\frac{b}{Q} \frac{\partial}{\partial t}+\frac{\rho_{22}}{Q} \frac{\partial^{2}}{\partial t^{2}}\right] \psi=0, \\
& {\left[\nabla^{2}-\frac{b}{Q} \frac{\partial}{\partial t}-\frac{\rho_{11}}{(\mu+K)} \frac{\partial^{2}}{\partial t^{2}}\right] H} \\
& -\frac{K}{(\mu+K)} \phi_{2}=0, \\
& {\left[\nabla^{2}-\frac{2 K}{\gamma}-\frac{\rho \jmath}{\gamma} \frac{\partial^{2}}{\partial t^{2}}\right] \phi_{2}+\frac{K}{\gamma} \nabla^{2} H=0,}
\end{aligned}
$$

Following Fung [11], a Galilean transformation

$$
x^{*}=x+U t, \quad z^{*}=z, \quad t^{*}=t,
$$

is introduced, then the boundary conditions would be independent of $t^{*}$ and assuming the dimensionless variables defined by ,

$$
\begin{aligned}
x^{\prime} & =\frac{x^{*}}{h}, \quad z^{\prime}=\frac{z^{*}}{h}, \quad \phi_{2}^{\prime}=\frac{\jmath}{h^{2}} \phi_{2}, \\
q^{\prime} & =\frac{q}{h^{2}}, \quad H^{\prime}=\frac{H}{h^{2}}, \quad \psi^{\prime}=\frac{\psi}{h^{2}}, \\
t_{i j}^{\prime} & =\frac{t_{i j}}{\lambda}, \quad m_{i j}^{\prime}=\frac{m_{i j}}{\lambda h}, \quad \sigma^{\prime}=\frac{\sigma}{\lambda}, \\
E^{\prime} & =\frac{E}{h^{2}}, \quad L^{\prime}=\frac{L}{h^{2}},
\end{aligned}
$$

where $h$ is a parameter having dimension of length, in equations (12)-(15) and applying the Fourier transform defined by ,

$$
\tilde{f}(\xi, z)=\int_{-\infty}^{\infty} f(x, z) e^{\imath \xi x} d x
$$

we get (after suppressing the primes),

$$
\begin{aligned}
& {\left[\frac{d^{2}}{d z^{2}}-\xi^{2}+\frac{\lambda_{1}^{2}}{\lambda_{0}^{2}}\right] \tilde{q}} \\
& +\left[\frac{Q}{\lambda_{0}^{2}}\left(\frac{d^{2}}{d z^{2}}-\xi^{2}\right)+\frac{\lambda_{2}^{2}}{\lambda_{0}^{2}}\right] \tilde{\psi}=0, \\
& {\left[\frac{d^{2}}{d z^{2}}-\xi^{2}+\frac{\lambda_{2}^{2}}{Q}\right] \tilde{q}} \\
& +\left[\frac{R}{Q}\left(\frac{d^{2}}{d z^{2}}-\xi^{2}\right)+\frac{\lambda_{3}^{2}}{Q}\right] \tilde{\psi}=0, \\
& {\left[\frac{d^{2}}{d z^{2}}-\xi^{2}+\frac{\lambda_{1}^{2}}{\lambda_{6}^{2}}\right] \tilde{H}-\frac{\lambda_{4}^{2}}{\lambda_{6}^{2}} \tilde{\phi}_{2}=0,} \\
& {\left[\frac{d^{2}}{d z^{2}}-\xi^{2}+\frac{\lambda_{5}^{2}}{\gamma}\right] \tilde{\phi}_{2}+\frac{K \jmath}{\rho}\left(\frac{d^{2}}{d z^{2}}-\xi^{2}\right) \tilde{H}=0,}
\end{aligned}
$$

where

$$
\begin{aligned}
& \lambda_{0}^{2}=\lambda+2 \mu+K, \quad \lambda_{1}^{2}=U^{2} \xi^{2} \rho_{11}+\imath \xi b h U, \\
& \lambda_{2}^{2}=U^{2} \xi^{2} \rho_{12}-\imath \xi b h U, U^{2} \xi^{2} \rho_{22}+\imath \xi b h U, \\
& \lambda_{4}^{2}=\frac{K h^{2}}{\jmath}, \quad \lambda_{5}^{2}=\rho \jmath U^{2} \xi^{2}-2 K h^{2} \\
& \lambda_{6}^{2}=\mu+K .
\end{aligned}
$$


Eliminating $\tilde{\psi}$ from equations (19) and (20) and $\tilde{\phi}_{2}$ from (21) and (22) respectively, we get

$$
\begin{aligned}
& {\left[\frac{d^{4}}{d z^{4}}+A_{1} \frac{d^{2}}{d z^{2}}+B_{1}\right] \tilde{q}=0,} \\
& {\left[\frac{d^{4}}{d z^{4}}+A_{2} \frac{d^{2}}{d z^{2}}+B_{2}\right] \tilde{H}=0,}
\end{aligned}
$$

where,

$$
\begin{aligned}
A_{1} & =\frac{\lambda_{7}^{2}}{\lambda_{8}^{2}}-2 \xi^{2} \\
A_{2} & =\frac{\lambda_{1}^{2}}{\lambda_{6}^{2}}+\frac{K \jmath}{\gamma} \frac{\lambda_{4}^{2}}{\lambda_{6}^{2}}+\frac{\lambda_{5}^{2}}{\gamma}-2 \xi^{2}, \\
B_{1} & =\xi^{4}-\xi^{2} \frac{\lambda_{7}^{2}}{\lambda_{8}^{2}}+\frac{\left(\lambda_{1}^{2} \lambda_{3}^{2}-\lambda_{2}^{4}\right)}{\lambda_{8}^{2}}, \\
B_{2} & =\xi^{4}-\xi^{2}\left(\frac{\lambda_{1}^{2}}{\lambda_{6}^{2}}+\frac{K J}{\gamma} \frac{\lambda_{4}^{2}}{\lambda_{6}^{2}}+\frac{\lambda_{5}^{2}}{\gamma}\right)+\frac{\lambda_{1}^{2}}{\lambda_{6}^{2}} \frac{\lambda_{5}^{2}}{\gamma} \\
\lambda_{7}^{2} & =R \lambda_{1}^{2}+\lambda_{0}^{2} \lambda_{3}^{2}-2 \lambda_{2}^{2} Q \\
\lambda_{8}^{2} & =R \lambda_{0}^{2}-Q^{2}
\end{aligned}
$$

The solutions of equations (24) and (25) with $A_{1}, A_{2}, B_{1}, B_{2}$ defined by equation (26) and satisfying the radiation conditions that $\tilde{q}, \tilde{\psi}, \tilde{H}, \tilde{\phi}_{2} \rightarrow 0$ as $z \rightarrow \infty$ are,

$$
\begin{aligned}
\tilde{q} & =D_{1} e^{-q_{1} z}+D_{2} e^{-q_{2} z}, \\
\tilde{\psi} & =a_{1} D_{1} e^{-q_{1} z}+a_{2} D_{2} e^{-q_{2} z}, \\
\tilde{H} & =D_{3} e^{-q_{3} z}+D_{4} e^{-q_{4} z}, \\
\tilde{\phi}_{2} & =a_{3} D_{3} e^{-q_{1} z}+a_{4} D_{4} e^{-q_{4} z},
\end{aligned}
$$

where, $q_{1,2}^{2}$ and $q_{3,4}^{2}$ are the roots of equations (24) and (25) respectively given by

$$
\begin{aligned}
& q_{1,2}^{2}=\frac{-A_{1} \pm \sqrt{\left(A_{1}^{2}-4 B_{1}\right)}}{2} \\
& q_{3,4}^{2}=\frac{-A_{2} \pm \sqrt{\left(A_{2}^{2}-4 B_{2}\right)}}{2}
\end{aligned}
$$

the coupling constants $a_{1}, a_{2}, a_{3}, a_{4}$ are defined by

$$
\begin{aligned}
& a_{1,2}=\frac{\lambda_{0}^{2}\left(\xi^{2}-q_{1,2}^{2}\right)-\lambda_{1}^{2}}{Q\left(q_{1,2}^{2}-\xi^{2}\right)+\lambda_{2}^{2}}, \\
& a_{3,4}=\frac{\lambda_{6}^{2}\left(q_{3,4}^{2}-\xi^{2}\right)+\lambda_{1}^{2}}{\lambda_{4}^{2}} .
\end{aligned}
$$

Adopting the same approach, we find the solutions for the elastic medium (Medium II) as,

$$
\begin{aligned}
\tilde{E} & =D_{5} e^{q_{5} z}, \\
\tilde{L} & =D_{6} e^{q_{6} z},
\end{aligned}
$$

where $E$ and $L$ are potential functions in medium II related to the displacement components $v_{1}$ and $v_{2}$ as,

$$
v_{1}=\frac{\partial E}{\partial x}+\frac{\partial L}{\partial z}, \quad v_{3}=\frac{\partial E}{\partial z}-\frac{\partial L}{\partial x},
$$

and

$$
q_{5}^{2}=\xi^{2}\left(1-\frac{\rho^{e} U^{2}}{\lambda^{e}+\mu^{e}}\right), \quad q_{6}^{2}=\xi^{2}\left(1-\frac{\rho^{e} U^{2}}{\mu^{e}}\right) .
$$

\section{Boundary Conditions}

For a concentrated point force, we take $P(x+U t)=F \delta\left(x^{*}\right)$ where $\delta\left(x^{*}\right)$ is Dirac-delta function and $F$ is the magnitude of force applied along the interface of two media. Therefore in moving coordinates the boundary conditions at the interface $z=0$ are,

$$
\begin{aligned}
\text { (i) } & t_{33}=t_{33}^{e}-F \delta\left(x^{*}\right), \\
\text { (ii) } & t_{31}=t_{33}^{e} \\
(\text { iii }) & u_{1}=v_{1} \\
\text { (iv) } & u_{3}=v_{3} \\
\text { (v) } & m_{32}=0 \\
\text { (vi }) & \sigma=0 .
\end{aligned}
$$

Using equations (4)-(6), (8), (11), (16), (17) and (34) inthe boundary conditions (36), we obtain the boundary conditions in the dimensionless form. On suppressing the primes and applying the Fourier transform defined by (18) in the dimensionless boundary conditions and using (27)-(30), (32) and (33) in the resulting transformed boundary conditions, we obtain the transformed expressions for displacement, microrotation, force stress and couple stress in micropolar liquid-saturated porous medium as,

$$
\begin{aligned}
& \tilde{u}_{1}=\frac{1}{\triangle}\left[l_{1} \triangle_{1} e^{-q_{1} z}+l_{2} \triangle_{2} e^{-q_{2} z}\right. \\
& \left.+l_{3} \triangle_{3} e^{-q_{3} z}+l_{4} \triangle_{4} e^{-q_{4} z}\right], \\
& \tilde{u}_{3}=\frac{1}{\triangle}\left[p_{1} \triangle_{1} e^{-q_{1} z}+p_{2} \triangle_{2} e^{-q_{2} z}\right. \\
& \left.+p_{3} \triangle_{3} e^{-q_{3} z}+p_{4} \triangle_{4} e^{-q_{4} z}\right], \\
& \tilde{\phi}_{2}=\frac{1}{\triangle}\left[a_{3} \triangle_{3} e^{-q_{3} z}+a_{4} \triangle_{4} e^{-q_{4} z}\right] \text {, } \\
& \tilde{t}_{31}=\frac{1}{\triangle}\left[s_{1} \triangle_{1} e^{-q_{1} z}+s_{2} \triangle_{2} e^{-q_{2} z}\right. \\
& \left.+s_{3} \triangle_{3} e^{-q_{3} z}+s_{4} \triangle_{4} e^{-q_{4} z}\right], \\
& \tilde{t}_{33}=\frac{1}{\triangle}\left[r_{1} \triangle_{1} e^{-q_{1} z}+r_{2} \triangle_{2} e^{-q_{2} z}\right. \\
& \left.+r_{3} \triangle_{3} e^{-q_{3} z}+r_{4} \triangle_{4} e^{-q_{4} z}\right], \\
& \tilde{m}_{32}=\frac{1}{\triangle}\left[b_{3} \triangle_{3} e^{-q_{3} z}+b_{4} \triangle_{4} e^{-q_{4} z}\right] \text {, }
\end{aligned}
$$




$$
\tilde{\sigma}=\frac{1}{\triangle}\left[n_{1} \triangle_{1} e^{-q_{1} z}+n_{2} \triangle_{2} e^{-q_{2} z}\right]
$$

where

$$
\begin{aligned}
& \triangle=-f_{1}^{\prime} \triangle_{0}+f_{2}^{\prime}\left[f_{1} g_{1}-f_{3} g_{4}+f_{4} g_{5}\right] \\
& -f_{3}^{\prime}\left[f_{1} g_{2}-f_{2} g_{4}+f_{4} g_{6}\right] \\
& +f_{4}^{\prime}\left[f_{1} g_{3}-f_{2} g_{5}+f_{3} g_{6}\right] \text {, } \\
& \triangle_{1}=-F n_{2} \triangle_{0}, \quad \triangle_{2}=F n_{1} \triangle_{0}, \\
& \triangle_{3}=-F b_{4} \triangle_{0}^{\prime}, \quad \triangle_{4}=F b_{3} \triangle_{0}^{\prime} \text {, } \\
& \triangle_{0}=f_{2} g_{1}-f_{3} g_{2}+f_{4} g_{3}, \\
& \triangle_{0}^{\prime}=f_{2}^{\prime} g_{1}-f_{3}^{\prime} g_{2}+f_{4}^{\prime} g_{3}, \\
& r_{1,2}=\left(\frac{\lambda_{0}^{2}+Q a_{1,2}}{\lambda}\right) q_{1,2}^{2}-\left(1+\frac{Q a_{1,2}}{\lambda}\right) \xi^{2}, \\
& r_{3,4}=-\imath \xi g_{0} q_{3,4}, \\
& r_{5}=\left(1+2 f_{0}\right) q_{5}^{2}-\xi^{2}, \\
& r_{6}=2 \imath \xi f_{0} q_{6} \text {, } \\
& f_{0}=\frac{\mu^{e}}{\lambda^{e}} \\
& g_{0}=\frac{2 \mu+K}{\lambda}, \\
& s_{1,2}=\imath \xi g_{0} q_{1,2}, \\
& s_{3,4}=\frac{\lambda_{6}^{2} q_{3,4}^{2}}{\lambda}+\frac{\mu \xi^{2}}{\lambda}-\frac{\lambda_{4}^{2} a_{4}}{\lambda}, \\
& s_{5}=-2 \imath \xi f_{0} q_{5}, \\
& s_{6}=f_{0}\left(q_{6}^{2}+\xi^{2}\right) \text {, } \\
& l_{1}=l_{2}=l_{5}=-\imath \xi \text {, } \\
& l_{3,4}=-q_{3,4}, \quad l_{6}=q_{6}, \\
& p_{1,2}=-q_{1,2}, \quad p_{5}=q_{5}, \\
& p_{3}=p_{4}=p_{5}=\imath \xi \text {, } \\
& b_{3,4}=-\frac{\gamma}{\jmath \lambda} a_{3,4} q_{3,4}, \\
& n_{1,2}=\left(\frac{Q+R a_{1,2}}{\lambda}\right)\left(q_{1,2}^{2}-\xi^{2}\right), \\
& f_{1}=b_{4} r_{3}-b_{3} r_{4}, \quad f_{2}=b_{4} s_{3}-b_{3} s_{4}, \\
& f_{3}=b_{4} l_{3}-b_{3} l_{4}, \quad f_{4}=b_{4} p_{3}-b_{3} p_{4}, \\
& f_{1}^{\prime}=n_{1} r_{2}-n_{2} r_{1}, \quad f_{2}^{\prime}=n_{1} s_{2}-n_{2} s_{1} \text {, } \\
& f_{3}^{\prime}={ }_{1} l_{2}-n_{2} l_{1}, \quad f_{4}^{\prime}=n_{1} p_{2}-n_{2} p_{1} \text {, } \\
& g_{1}=l_{5} p_{6}-p_{5} l_{6}, \quad g_{2}=s_{5} p_{6}-p_{5} s_{6}, \\
& g_{3}=s_{5} l_{6}-l_{5} s_{6}, \quad g_{4}=r_{5} p_{6}-p_{5} r_{6} \text {, } \\
& g_{5}=r_{5} l_{6}-l_{5} r_{6}, \quad g_{6}=r_{5} s_{6}-s_{5} r_{6} \text {. }
\end{aligned}
$$

\section{Particular Cases}

6.1 Neglecting Porous effect in Medium I (i.e. $Q=R=b=0$ in equations (1), (2), (4) and (6)), the problem reduces to moving load at the interface of elastic half space and micropolar elastic half space (Kumar \&
Ailawalia [22]). The transformed expressions for displacement, microrotation, force stress, couple stress in micropolar elastic medium in this case are given by,

$$
\begin{aligned}
& \tilde{u}_{1}=\frac{1}{\triangle^{(1)}}\left[l_{1}^{\prime} \triangle_{1}^{(1)} e^{-q_{1}^{\prime} z}\right. \\
& \left.+l_{3}^{\prime} \triangle_{3}^{(1)} e^{-q_{3}^{\prime} z}+l_{4}^{\prime} \triangle_{4}^{(1)} e^{-q_{4}^{\prime} z}\right], \\
& \tilde{u}_{3}=\frac{1}{\triangle^{(1)}}\left[p_{1}^{\prime} \triangle_{1}^{(1)} e^{-q_{1}^{\prime} z}\right. \\
& \left.+p_{3}^{\prime} \triangle_{3}^{(1)} e^{-q_{3}^{\prime} z}+p_{4}^{\prime} \triangle_{4}^{(1)} e^{-q_{4}^{\prime} z}\right], \\
& \tilde{\phi}_{2}=-\frac{\gamma}{\jmath \lambda \triangle^{(1)}}\left[a_{5} \triangle_{3}^{(1)} e^{-q_{3}^{\prime} z}+a_{6} \triangle_{4}^{(1)} e^{-q_{4}^{\prime} z}\right] \text {, } \\
& \tilde{t}_{31}=\frac{1}{\triangle^{(1)}}\left[s_{1}^{\prime} \triangle_{1}^{(1)} e^{-q_{1}^{\prime} z}\right. \\
& \left.+s_{3}^{\prime} \triangle_{3}^{(1)} e^{-q_{3}^{\prime} z}+s_{4}^{\prime} \triangle_{4}^{(1)} e^{-q_{4}^{\prime} z}\right], \\
& \tilde{t}_{33}=\frac{1}{\triangle^{(1)}}\left[r_{1}^{\prime} \triangle_{1}^{(1)} e^{-q_{1}^{\prime} z}\right. \\
& \left.+r_{3}^{\prime} \triangle_{3}^{(1)} e^{-q_{3}^{\prime} z}+r_{4}^{\prime} \triangle_{4}^{(1)} e^{-q_{4}^{\prime} z}\right] \\
& \tilde{m}_{32}=\frac{1}{\triangle^{(1)}}\left[b_{3}^{\prime} \triangle_{3}^{(1)} e^{-q_{3}^{\prime} z}+b_{4}^{\prime} \triangle_{4}^{(1)} e^{-q_{4}^{\prime} z}\right],
\end{aligned}
$$

where,

$$
\begin{aligned}
& \triangle^{(1)}= s_{1}^{\prime}\left(M_{1} g_{1}-M_{3} g_{4}+M_{4} g_{5}\right) \\
&+l_{1}^{\prime}\left(M_{2} g_{4}-M_{1} g_{2}+M_{4} g_{6}\right) \\
& \quad+p_{1}^{\prime}\left(M_{1} g_{3}-M_{2} g_{5}+M_{3} g_{6}\right)-\frac{r \triangle_{1}^{(1)}}{F}, \\
& \triangle_{1}^{(1)}=-F b_{3}^{\prime}\left(M_{3} g_{2}-M_{2} g_{1}+M_{4} g_{3}\right), \\
& \triangle_{3}^{(1)}= \frac{b_{4}^{\prime}}{b_{3}^{\prime}} \triangle_{4}^{(1)}, \\
& \triangle_{4}^{(1)}=-F b_{3}^{\prime}\left(s_{1}^{\prime} g_{1}-l_{1}^{\prime} g_{2}+p_{1}^{\prime} g_{3}\right), \\
& q_{1}^{\prime}= \xi^{2}\left(1-\frac{\rho_{11} U^{2}}{\lambda_{0}^{2}}\right), \\
& q_{3,4}^{\prime}= \frac{-A_{2}^{\prime} \pm \sqrt{A_{2}^{\prime 2}-4 B_{2}^{\prime}}}{2}, \\
& a_{5,6}= {\left[\frac{\lambda_{6}^{2}\left(q_{3,4}^{\prime}-\xi^{2}\right)+k_{1}^{2}}{\lambda_{4}^{2}}\right], } \\
& A_{2}^{\prime}= \frac{k_{1}^{2}}{\lambda_{6}^{2}}-\frac{2 \jmath \lambda_{4}^{2}}{\gamma}+\frac{k_{2}^{2}}{\gamma}+\frac{K \jmath}{\gamma} \frac{\lambda_{4}^{2}}{\lambda_{6}^{2}}-2 \xi^{2}, \\
& B_{2}^{\prime}= \xi^{4}-\xi^{2} A_{2}^{\prime}-\frac{2 \jmath}{\gamma \lambda_{2}^{2} \lambda_{4}^{2}}+\frac{k_{1}^{2} k_{2}^{2}}{\gamma \lambda_{6}^{2}}, \\
& k_{1}^{2}= \rho_{11} U^{2} \xi^{2}, \\
& r_{1}^{\prime}= \frac{\lambda_{0}^{2} q_{1}^{\prime}}{\lambda}-\xi_{2}^{2}, \rho \jmath U^{2} \xi^{2}, \\
& k_{3,4}^{2}=-\imath \xi g_{0} q_{3,4}^{\prime},
\end{aligned}
$$




$$
\begin{gathered}
s_{1}^{\prime}=\imath \xi g_{0} q_{1}^{\prime}, \quad l_{1}^{\prime}=l_{5}^{\prime}=-\imath \xi, \\
l_{3,4}^{\prime}=-q_{3,4}^{\prime}, \quad p_{1}^{\prime}=-q_{1}^{\prime}, \\
p_{3}^{\prime}=p_{4}^{\prime}=\imath \xi, \quad b_{3,4}^{\prime}=-\frac{\gamma a_{5,6} q_{3,4}^{\prime}}{\jmath \lambda}, \\
M_{1}=b_{3}^{\prime} r_{4}^{\prime}-b_{4}^{\prime} r_{3}^{\prime}, \quad M_{2}=b_{3}^{\prime} s_{4}^{\prime}-b_{4}^{\prime} s_{3}^{\prime}, \\
M_{3}=b_{3}^{\prime} l_{4}^{\prime}-b_{4}^{\prime} l_{3}^{\prime}, \quad M_{4}=b_{3}^{\prime} p_{4}^{\prime}-b_{4}^{\prime} p_{3}^{\prime} .
\end{gathered}
$$

6.2 Neglecting both micropolarity effect and porous effect in medium I (i.e. $\alpha=\beta=K=\jmath=Q=R=$ $b=0$ in equations (1)-(6), the problem reduces to steady state response at the interface of two elastic half spaces with different properties. The transformed expressions for displacements and force stress in elastic medium (Medium I) reduce to,

$$
\begin{aligned}
\tilde{u}_{1} & =\frac{1}{\triangle^{(2)}}\left[l_{1}^{\prime \prime} \triangle_{1}^{(2)} e^{-q_{1}^{\prime \prime} z}+l_{3}^{\prime \prime} \triangle_{3}^{(2)} e^{-q_{3}^{\prime \prime} z}\right], \\
\tilde{u}_{3} & =\frac{1}{\triangle^{(2)}}\left[p_{1}^{\prime \prime} \triangle_{1}^{(2)} e^{-q_{1}^{\prime \prime} z}+p_{3}^{\prime \prime} \triangle_{3}^{(2)} e^{-q_{3}^{\prime \prime} z}\right], \\
\tilde{t}_{31} & =\frac{1}{\triangle^{(2)}}\left[s_{1}^{\prime \prime} \triangle_{1}^{(2)} e^{-q_{1}^{\prime \prime} z}+s_{3}^{\prime \prime} \triangle_{3}^{(2)} e^{-q_{3}^{\prime \prime} z}\right], \\
\tilde{t}_{33} & =\frac{1}{\triangle^{(2)}}\left[r_{1}^{\prime \prime} \triangle_{1}^{(2)} e^{-q_{1}^{\prime \prime} z}+r_{3}^{\prime \prime} \triangle_{3}^{(2)} e^{-q_{3}^{\prime \prime} z}\right],
\end{aligned}
$$

where,

$$
\begin{aligned}
& \triangle^{(2)}=- \frac{r_{1}^{\prime \prime}}{F} \triangle_{1}^{(2)}+s_{1}^{\prime \prime}\left[l_{3}^{\prime \prime} g_{4}-r_{3}^{\prime \prime} g_{1}-p_{3}^{\prime \prime} g_{5}\right] \\
& \quad+l_{1}^{\prime \prime}\left[r_{3}^{\prime \prime} g_{2}+p_{3}^{\prime \prime} g_{6}-s_{3}^{\prime \prime} g_{4}\right] \\
& \quad-p_{1}^{\prime \prime}\left[r_{3}^{\prime \prime} g_{3}+l_{3}^{\prime \prime} g_{6}-s_{3}^{\prime \prime} g_{5}\right], \\
& \triangle_{1}^{(2)}=-F\left[s_{3}^{\prime \prime} g_{1}-l_{3}^{\prime \prime} g_{2}+p_{3}^{\prime \prime} g_{3}\right], \\
& \triangle_{3}^{(2)}=F\left[s_{1}^{\prime \prime} g_{1}-l_{1}^{\prime \prime} g_{2}+p_{1}^{\prime \prime} g_{3}\right], \\
& q_{1}^{\prime \prime 2}=\xi^{2}\left(1-\frac{\rho_{11} U^{2}}{\lambda+2 \mu}\right), \quad q_{3}^{\prime \prime} 2=\xi^{2}\left(1-\frac{\rho_{11} U^{2}}{\mu}\right), \\
& r_{1}^{\prime \prime}=\left(\frac{\lambda+\mu}{\lambda}\right) q_{1}^{\prime \prime 2}-\xi^{2}, \quad r_{3}^{\prime \prime}=-2 \imath \xi \frac{\mu}{\lambda} q_{3}^{\prime \prime} 2 \\
& s_{1}^{\prime \prime}=2 \imath \xi \frac{\mu}{\lambda} q_{1}^{\prime \prime 2}, \quad s_{3}^{\prime \prime}=\frac{\mu}{\lambda}\left(q_{3}^{\prime \prime 2}+\xi^{2}\right), \\
& l_{1}^{\prime \prime}=-\imath \xi, \quad l_{3}^{\prime \prime}=-q_{3}^{\prime \prime}, \\
& p_{1}^{\prime \prime}=-q_{1}^{\prime \prime}, \quad p_{3}^{\prime \prime}=\imath \xi .
\end{aligned}
$$

6.3 Neglecting porous effect in Medium I (i.e. $Q=R=b=0$ in equations (1), (2), (4) and (6)), and letting $\mu^{e} \rightarrow 0$ in Medium II i.e. in equations (7) and (8), we obtain the expressions for displacement, microrotation, force stress, couple stress in micropolar medium (medium I) at non-viscous fluid/micropolar solid interface as,

$$
\begin{aligned}
\tilde{u}_{1}=\frac{1}{\triangle^{(3)}} & {\left[l_{1}^{\prime} \triangle_{1}^{(3)} e^{-q_{1}^{\prime} z}\right.} \\
& \left.+l_{3}^{\prime} \triangle_{3}^{(3)} e^{-q_{3}^{\prime} z}+l_{4}^{\prime} \triangle_{4}^{(3)} e^{-q_{4}^{\prime} z}\right]
\end{aligned}
$$

$$
\begin{aligned}
\tilde{u}_{3}= & \frac{1}{\triangle^{(3)}}\left[p_{1}^{\prime} \triangle_{1}^{(3)} e^{-q_{1}^{\prime} z}\right. \\
& \left.+p_{3}^{\prime} \triangle_{3}^{(3)} e^{-q_{3}^{\prime} z}+p_{4}^{\prime} \triangle_{4}^{(3)} e^{-q_{4}^{\prime} z}\right], \\
\tilde{\phi}_{2}= & -\frac{\gamma}{j \lambda \triangle^{(3)}}\left[a_{5} \triangle_{3}^{(3)} e^{-q_{3}^{\prime} z}+a_{6} \triangle_{4}^{(3)} e^{-q_{4}^{\prime} z}\right], \\
\tilde{t}_{31}= & \frac{1}{\triangle^{(3)}}\left[s_{1}^{\prime} \triangle_{1}^{(3)} e^{-q_{1}^{\prime} z}\right. \\
& \left.+s_{3}^{\prime} \triangle_{3}^{(3)} e^{-q_{3}^{\prime} z}+s_{4}^{\prime} \triangle_{4}^{(3)} e^{-q_{4}^{\prime} z}\right], \\
\tilde{t}_{33}= & \frac{1}{\triangle^{(3)}}\left[r_{1}^{\prime} \triangle_{1}^{(3)} e^{-q_{1}^{\prime} z}\right. \\
& \left.+r_{3}^{\prime} \triangle_{3}^{(3)} e^{-q_{3}^{\prime} z}+r_{4}^{\prime} \triangle_{4}^{(3)} e^{-q_{4}^{\prime} z}\right], \\
\tilde{m}_{32}= & \frac{1}{\triangle^{(3)}}\left[b_{3}^{\prime} \triangle_{3}^{(3)} e^{-q_{3}^{\prime} z}+b_{4}^{\prime} \triangle_{4}^{(3)} e^{-q_{4}^{\prime} z}\right],
\end{aligned}
$$

where,

$$
\begin{aligned}
\triangle^{(3)} & =s_{1}^{\prime} p_{5}^{\prime} M_{1}+\left(p_{1}^{\prime} r_{5}^{\prime}-r_{1}^{\prime} p_{5}^{\prime}\right) M_{2}-s_{1}^{\prime} r_{5}^{\prime} M_{4}, \\
\triangle_{1}^{(3)} & =F p_{5}^{\prime} M_{2}, \quad \triangle_{3}^{(3)}=\frac{b_{4}^{\prime} \triangle_{4}^{(3)}}{b_{3}^{\prime}}, \\
\triangle_{4}^{(3)} & =-F b_{3}^{\prime} s_{1}^{\prime} p_{5}^{\prime}, \quad q_{5}^{\prime}=\xi^{2}\left(1-\frac{\rho U^{2}}{\lambda^{e}}\right), \\
r_{5}^{\prime} & =q_{5}^{\prime}-\xi^{2}, \quad p_{5}^{\prime}=q_{5}^{\prime} .
\end{aligned}
$$

Kumar and Ailawalia [23] have obtained these expressions for subsonic, supersonic and transonic load velocities.

6.4 Neglecting porous effect in Medium I (i.e. $Q=R=b=0$ in equations (1), (2), (4) and (6)) and letting $\lambda^{e}, \mu^{e} \rightarrow 0$ in Medium II, i.e. in equations (7), (8), we obtain the expressions for displacement, microrotation, force stress and couple stress in micropolar elastic solid due to a moving load at the free surface (Sengupta and Ghosh [32]) as,

$$
\begin{gathered}
\tilde{u}_{1}=\frac{1}{\triangle^{(4)}}\left[l_{1}^{\prime} \triangle_{1}^{(4)} e^{-q_{1}^{\prime} z}\right. \\
\left.+l_{3}^{\prime} \triangle_{3}^{(4)} e^{-q_{3}^{\prime} z}+l_{4}^{\prime} \triangle_{4}^{(4)} e^{-q_{4}^{\prime} z}\right], \\
\tilde{u}_{3}=\frac{1}{\triangle^{(4)}}\left[p_{1}^{\prime} \triangle_{1}^{(4)} e^{-q_{1}^{\prime} z}\right. \\
\left.\quad+p_{3}^{\prime} \triangle_{3}^{(4)} e^{-q_{3}^{\prime} z}+p_{4}^{\prime} \triangle_{4}^{(4)} e^{-q_{4}^{\prime} z}\right], \\
\tilde{\phi}_{2}=-\frac{\gamma}{j \lambda \triangle^{(4)}}\left[a_{5} \triangle_{3}^{(4)} e^{-q_{3}^{\prime} z}+a_{6} \triangle_{4}^{(4)} e^{-q_{4}^{\prime} z}\right], \\
\tilde{t}_{31}=\frac{1}{\triangle^{(4)}}\left[s_{1}^{\prime} \triangle_{1}^{(4)} e^{-q_{1}^{\prime} z}\right. \\
\left.\quad+s_{3}^{\prime} \triangle_{3}^{(4)} e^{-q_{3}^{\prime} z}+s_{4}^{\prime} \triangle_{4}^{(4)} e^{-q_{4}^{\prime} z}\right] \\
\tilde{t}_{33}=\frac{1}{\triangle^{(4)}}\left[r_{1}^{\prime} \triangle_{1}^{(4)} e^{-q_{1}^{\prime} z}\right. \\
\left.\quad+r_{3}^{\prime} \triangle_{3}^{(4)} e^{-q_{3}^{\prime} z}+r_{4}^{\prime} \triangle_{4}^{(4)} e^{-q_{4}^{\prime} z}\right] \\
\frac{1}{\triangle^{(4)}}\left[b_{3}^{\prime} \triangle_{3}^{(4)} e^{-q_{3}^{\prime} z}+b_{4}^{\prime} \triangle_{4}^{(4)} e^{-q_{4}^{\prime} z}\right],
\end{gathered}
$$


where,

$$
\begin{aligned}
& \triangle^{(4)}=-\left(s_{1}^{\prime} M_{1}+r_{1}^{\prime} M_{2}\right), \quad \triangle_{1}^{(4)}=F M_{2}, \\
& \triangle_{3}^{(4)}=F s_{1}^{\prime} b_{4}^{\prime}, \quad \triangle_{4}^{(4)}=-\frac{-b_{3}^{\prime} \triangle_{3}^{(4)}}{b_{4}^{\prime}} .
\end{aligned}
$$

\section{Inversion of the Transform}

To obtain the solution of the problem in the physical domain, we invert the transform in (37)-(43), (45)-(50), (52)-(55), (56)-(62) and (64)-(69). These expressions are functions of $z$ and the parameter of Fourier transform $\xi$, hence are of the form $\tilde{f}(\xi, z)$. To get the function $f(x, z)$ in the physical domain we invert the Fourier transform using,

$$
\begin{aligned}
f(x, z) & =\frac{1}{2 \pi} \int_{-\infty}^{\infty} \tilde{f}(\xi, z) e^{-\imath \xi x} d z \\
& =\frac{1}{\pi} \int_{-\infty}^{\infty}\left(\cos (\xi x) f_{e}-\imath \sin (\xi x) f_{0}\right) d z
\end{aligned}
$$

where $f_{e}$ and $f_{0}$ are respectively even and odd parts of the function $\tilde{f}(\xi, z)$. The method for evaluating this integral is described by Press et al.[30] which involves the use of Rhomberg's integration with adaptive step size. This also uses the results from successive refinements of the extended trapezoidal rule followed by extrapolation of the results to the limit when the step size tends to zero.

\section{Numerical Results and Discussions}

Following Gauthier [12], we take following values of the relevant micropolar constants as

$$
\begin{aligned}
\rho & =2.19 \mathrm{gm} / \mathrm{cm}^{3}, \\
\gamma & =0.268 \times 10^{11} \text { dyne, } \\
\jmath & =0.196 \mathrm{~cm}^{2}, \\
K & =0.0149 \times 10^{11} \mathrm{dyne} / \mathrm{cm}^{2},
\end{aligned}
$$

Following Yew and Jogi [34] and Fatt [9], the following values of relevant parameters have been taken for

\section{(i) Kerosene-Saturated Sandstone}

$$
\begin{aligned}
\rho_{11} & =1.926137 \mathrm{gm} / \mathrm{cm}^{3}, \\
Q & =0.07635 \times 10^{11} \mathrm{dyne} / \mathrm{cm}^{2}, \\
\rho_{12} & =-0.002137 \mathrm{gm} / \mathrm{cm}^{3}, \\
R & =0.0326 \times 10^{11} \mathrm{dyne} / \mathrm{cm}^{2}, \\
\rho_{22} & =0.215337 \mathrm{gm} / \mathrm{cm}^{3}, \\
\lambda & =0.4339 \times 10^{11} \mathrm{dyne} / \mathrm{cm}^{2}, \\
\mu & =0.2765 \times 10^{11} \mathrm{dyne} / \mathrm{cm}^{2} .
\end{aligned}
$$

\section{(ii) Water-Saturated Sandstone}

$$
\begin{aligned}
\rho_{11} & =1.9032 \mathrm{gm} / \mathrm{cm}^{3}, \\
Q & =0.013 \times 10^{11} \text { dyne } / \mathrm{cm}^{2}, \\
\rho_{12} & =0 \mathrm{gm} / \mathrm{cm}^{3}, \\
R & =0.0637 \times 10^{11} \mathrm{dyne} / \mathrm{cm}^{2}, \\
\rho_{22} & =0.268 \mathrm{gm} / \mathrm{cm}^{3}, \\
\lambda & =0.306 \times 10^{11} \mathrm{dyne} / \mathrm{cm}^{2}, \\
\mu & =0.922 \times 10^{11} \text { dyne } / \mathrm{cm}^{2} .
\end{aligned}
$$

The Lame's constants $\left(\lambda^{e}, \mu^{e}\right)$ and density $\rho^{e}$ for elastic medium (Medium II) are given by Love [27],

$$
\begin{aligned}
& \lambda^{e}=2.4 \times 10^{11} \text { dyne } / \mathrm{cm}^{2}, \\
& \mu^{e}=1.2 \times 10^{11} \text { dyne } / \mathrm{cm}^{2}, \\
& \rho^{e}=1.2 \mathrm{gm} / \mathrm{cm}^{3} .
\end{aligned}
$$

The variations of normal displacement $u_{3}$, normal force stress $t_{33}$, tangential couple stress $m_{32}$ and normal stress in fluid $\sigma$ with horizontal distance at the plane $z=0.1$ and $z=1.0$ and $h=1.0 \mathrm{~cm}$ for

(i) Micropolar liquid-saturated porous medium (MPES) are shown by solid line at $z=0.1$ and dashed line at $z=1.0$.

(ii) Micropolar elastic solid (MES) are shown by centered symbol $(*)$ at $z=0.1$ and dashed line with centered symbol (*) at $z=1.0$.

(iii) Elastic solid (ES) are shown by solid line with centered symbol $(\odot)$ at $z=0.1$ and dashed line with centered symbol $(\bigodot)$ at $z=1.0$.

These variations are shown in figures (2)-(5)

\section{Discussions for Various Cases}

The values of normal displacement for micropolar theory of elasticity i.e. MPES and MES lie in a very short range. These values for a particular medium are quite close to each other at both the planes $z=0.1$ and $z=1.0$. As far as classical theory of elasticity is concerned, the values of normal displacement are more as compared to the values obtained for micropolar theory. In this case also the values are close at both the planes. These variations of normal displacement are shown in figure 2.

It is observed from figure 3, that the values of normal force stress increase sharply in the range $0 \leq x \leq 2.0$ and oscillate in the remaining range. Similar to the case discussed for normal displacement, the values of normal force stress obtained for micropolar theory of elasticity (MPES and MES) are of comparable magnitude in comparison to the classical theory. Also there is not much difference in the values obtained at two different planes i.e. $z=0.1$ and $z=1.0$. 


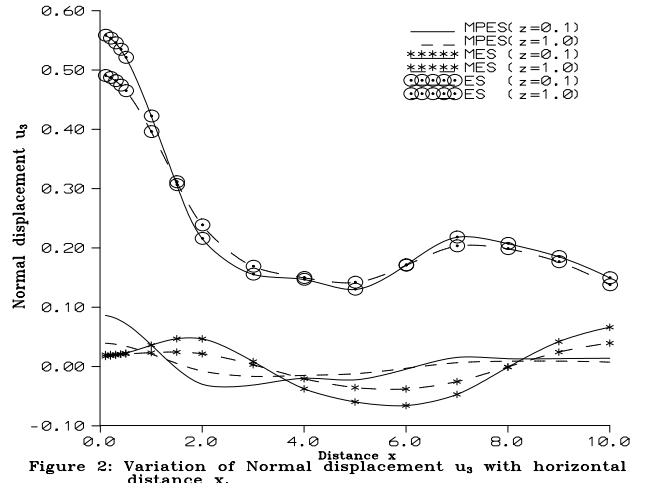

Figure 2: Variation of Normal Diftance $\begin{aligned} & \text { displacement } \\ & \text { distance } x \text {. }\end{aligned}$

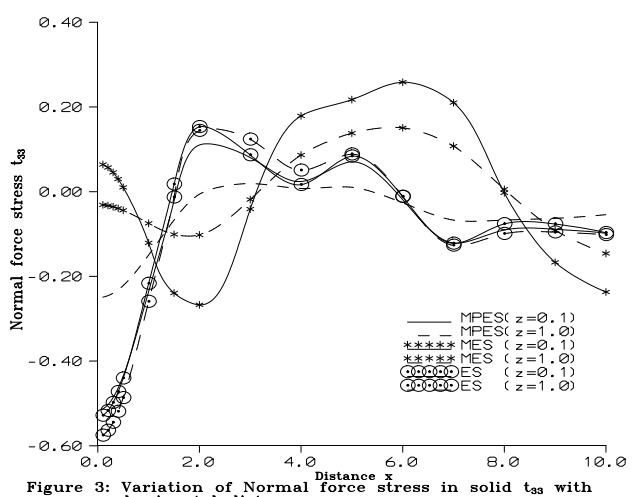

Contrary to the variations of normal displacement and normal force stress, the variations of tangential couple stress for both MPES and MES are more oscillatory at $z=1.0$ or we may also say that the values at the plane $z=0.1$ lies in the range -0.1 to +0.1 . These variations of tangential couple stress are shown in figure 4. Figure 5 shows that the difference in the values of normal stress in the liquid increase with the depth of plane considered (i.e. the value of $z$ ).

\section{Conclusion}

Micropolarity and porous effect play a very important role in the study of deformation of a body. Although there is not much difference in the values of normal displacement and normal force stress obtained at two planes, but this difference becomes significant, if we consider the values at different planes. The significant effect at different planes is however shown in the variations of tangential couple stress and normal stress in liquid part.
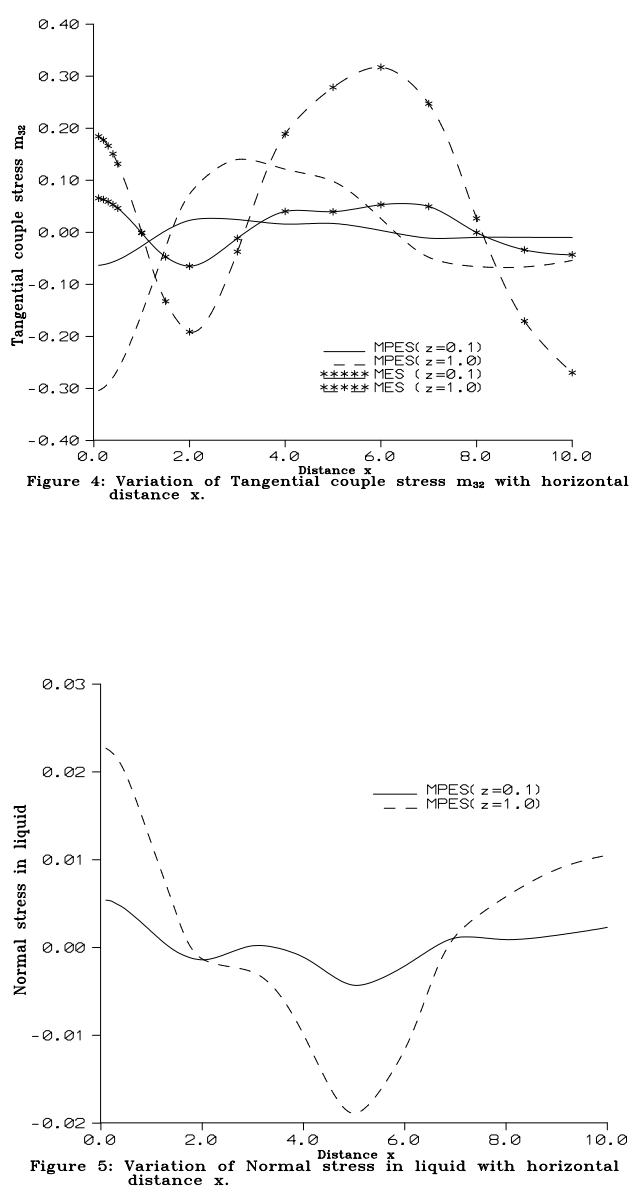

\section{References}

[1] Cole, J. and Huth, J., Stresses produced in a half space by moving loads. J. Appl. Mech. ASME, 25, 433-436 (1958).

[2] Deresiewicz, H., Mechanics of Granular Matter, in advances in Appl. Math, vol. V (edited by H. L. Dryden and T. H. Von Karma) Academic Press Inc., New York, U.S.A., 233-303 (1958).

[3] Deswal, S., Tomar, S. K. and Kumar, R., Effects of fluid viscosity on wave propagation in a cylindrical bore in micropolar elastic medium. Sadhana, 25, 439-452 (2000).

[4] Eason, G., The Stresses produced in a semi-infinite solid by moving surface force. Int. J. Engg. Sci., 2, 581-609 (1965).

[5] Eringen, A. C. and Suhubi, E. S., Non linear theory of simple microelastic solids I. Int. J. Engg. Sci., 2, 189-203 (1964).

[6] Eringen, A. C. and Suhubi, E. S., Non linear theory of simple microelastic solids II. Int. J. Engg. Sci., 2, 389-404 (1964).

[7] Eringen, A. C., Linear theory of micropolar elasticity. $J$. Math. Mech., 5, 909-923 (1966). 
[8] Ewing, W. M., Jardetzky, W. S. and Press, F., Elastic waves in layered media, Mcgraw Hill, (1957).

[9] Fatt, I., The Biot-wills elastic coefficients for a sand stone, J. Appl. Mech., 26, 296-297 (1959).

[10] Fryba, L., Vibration of solids and structures under moving loads. Thomas Telford, London, (1999).

[11] Fung, Y. C., Foundations of solid mechanics, Prentice Hall, New Delhi, (1968).

[12] Gauthier, R. D., Mechanics of micropolar media (Ed. O. Brulin and R. K. T. Hsieh), World scientific, Singapore, (1982).

[13] Ghosh, B. C., Steady state response to moving loads in the micropolar solid media. Proc., Indian, Natn., Sci. Acad., 51, 586-597 (1985).

[14] Konczak, Z., Thermo-mechanical effects in fluid-saturated porous media with a micropolar viscoelastic skelton. $Z$. Angew. Math. Mech., 66, 152-154 (1986).

[15] Konczak, Z., On Wave propagation in fluid-saturated porous media with micropolar viscoelastic skelton. Z. Angew. Math. Mech., 67, 201-203 (1987).

[16] Kumar, R. and Gogna, M. L., Steady State response to moving loads in micropolar elastic medium with stretch. Int. J. Engg. Sci., 30, 811-820 (1992).

[17] Kumar, R. and Miglani, A., Effects of pore Alignment on surface wave propagation in a liquid-saturated porous layer over a liquid-saturated porous half space with loosely bonded interface, J., Phy. Earth, 44, 153-172 (1996).

[18] Kumar, R. and Deswal, S., Steady State response of a micropolar generalized thermoelastic half-space to the moving mechanical/ thermal loads. Proc. Indian. Acad. Sci. (Math. Sci.), 110, 449-465 (2000).

[19] Kumar, R. and Deswal, S., Wave propagation in micropolar liquid saturated porous solid. Indian J. pure Appl. Math., 31, 1317-1337 (2000).

[20] Kumar, R. and Deswal, S., Steady State response to moving loads in a micropolar generalized thermoelastic half-space without energy dissipation. Ganita, 53, 23-42 (2002).

[21] Kumar, R. and Ailawalia, P., Moving load response at thermal conducting fluid and micropolar solid interface. Int. J. Appl. Mech. Engg., 8, 621-636 (2003).

[22] Kumar, R. and Ailawalia, P., Response to moving load at elastic/micropolar solid interface. South East Asian J. Math., Math. Sci., 2, 67-82 (2004).

[23] Kumar, R. and Ailawalia, P., Steady State response to moving load at fluid /micropolar solid interface. Seminar I. Vekua Inst. Appl. Math. REPORTS, 30, 6-16 (2004).

[24] Kumar, R. and Ailawalia, P., Effects of Viscosity with moving load at micropolar boundary surface. Int. J. Appl. Mech. Engg., 10, 95-108 (2005).

[25] Kumar, R. and Ailawalia, P., Moving load response in micropolar thermoelastic medium without energy dissipation possessing cubic symmetry, Int. J. Sol. Str., 44, 4068-4078 (2007).

[26] Kumar, R. and Barak M., Wave Propagation in liquidsaturated porous solid with micropolar elastic skelton at boundary surface. Appl. Math. Mech., 28, 337-349 (2007).

[27] Love, A. E. H., A treatise on the mathematical theory of elasticity, Dover, New York, (1944).

[28] Murad, M. A. and Cushman, J. H., Thermomechanical theories for swelling porous media with micro-structure. Int. J. Engg. Sci., 38, 517-564 (2000).
[29] Palmov, V. A., Fundamental equations of the theory of asymmetric elasticity. Prikl. Mat. Mekh. (in Russian), 28, 401-408 (1964).

[30] Payton R. G., Transient motion of an elastic half-space due to a moving surface line load. Int. J. Engg. Sci., 5, 49-79 (1967).

[31] Press, W. H, Teukolsky, S. A., Vellerling, W. T and Flannery, B.P., Numerical Recipes, (Cambridge: Cambridge University Press), (1986).

[32] Sengupta, P. R. and Ghosh, B. C., Waves and vibration in micropolar elastic medium I, Arch. Mech. Stos., 29, 273287 (1977).

[33] Sneddon, I. N., Fourier transforms, New York, McGrawHill, 445, (1951).

[34] Yew, C. H. and Jogi, P. N., Study of motion in fluid saturated porous rocks. J. Acoust. Soc. Am., 60, 2-8 (1976).

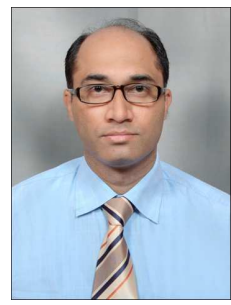

Praveen Ailawalia is presently employed as Professor at Baddi University of Emerging Sciences and Technology, Baddi, Solan, H.P., India. He obtained his Ph.D. from Kurukshetra University (India). He is an active researcher in the field of Thermoelasticity, Micropolar Elasticity with 15 years of teaching experience in various Universities and Colleges. He has published more than 65 (sixty five) research articles in reputed international journals and guided three research students for Ph.D. till date. He is also research reviewer of many International journals. 